\title{
Genetic and therapeutic targeting of properdin in mice prevents complement-mediated tissue injury
}

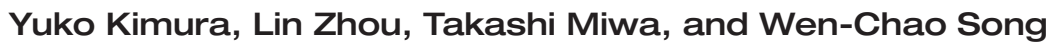 \\ Institute for Translational Medicine and Therapeutics and Department of Pharmacology, \\ University of Pennsylvania School of Medicine, Philadelphia, Pennsylvania, USA.
}

\begin{abstract}
The alternative pathway (AP) of complement activation is constitutively active and must be regulated by host proteins to prevent autologous tissue injury. Dysfunction of AP regulatory proteins has been linked to several human inflammatory disorders. Properdin is a positive regulator of AP complement activation that has been shown to extend the half-life of cell surface-bound $\mathrm{C} 3$ convertase $\mathrm{C} 3 \mathrm{bBb}$; it may also initiate AP complement activation. Here, we demonstrate a critical role for properdin in autologous tissue injury mediated by AP complement activation. We identified myeloid lineage cells as the principal source of plasma properdin by generating mice with global and tissue-specific knockout of $C f p$ (which encodes properdin) and by generating BM chimeric mice. Properdin deficiency rescued mice from AP complement-mediated embryonic lethality caused by deficiency of the membrane complement regulator Crry and markedly reduced disease severity in the $\mathrm{K} / \mathrm{BxN}$ model of arthritis. Ab neutralization of properdin in WT mice similarly ameliorated arthritis development, whereas reconstitution of properdin-null mice with exogenous properdin restored arthritis sensitivity. These data implicate systemic properdin as a key contributor to AP complement-mediated injury and support its therapeutic targeting in complement-dependent human diseases.
\end{abstract}

\section{Introduction}

The complement system is an important part of innate immunity that plays a vital role in host defense against opportunistic infections $(1,2)$. Activated complement promotes inflammation by anaphylatoxin generation, facilitates phagocytosis by target opsonization, and causes direct cell lysis by membrane attack complex formation $(1,2)$. Activation of the complement system occurs via 3 different pathways: the classical pathway, which is triggered by antigen-bound Abs; the lectin pathway, which is mediated by mannose-binding lectins with specificity to unique sugar molecules present on the surface of microbes; and the alternative pathway (AP), which is constantly active at a low level as a result of spontaneous hydrolysis of the complement protein C3 $(3,4)$. Among these activation mechanisms, the AP plays a pivotal role because of its self-amplifying nature and the fact that all pathways converge at the $\mathrm{C} 3$ cleavage step, from which the self-amplification loop of the AP can be engaged $(5,6)$.

Although Abs and collectins afford specificity to the classical and lectin pathways in recognizing non-self, avoidance of autologous tissue injury from the constitutively active and nondiscriminatory AP complement is achieved through host cell-specific complement regulatory proteins. Numerous human disorders have been linked to inadequate AP complement control, often as a consequence of regulator dysfunction $(2,7)$. For example, deficiency of decay-accelerating factor (DAF) and CD59 on blood cells causes paroxysmal nocturnal hemoglobinuria (PNH), for which eculizumab, a first-in-class anti-complement $\mathrm{C} 5 \mathrm{mAb}$ drug, has been developed as a treatment $(8,9)$. Atypical hemolytic uremic syndrome (aHUS) and age-related macular degeneration (AMD)

Conflict of interest: The authors have declared that no conflict of interest exists. Citation for this article: J Clin Invest. 2010;120(10):3545-3554. doi:10.1172/JCI41782. are 2 other conditions caused by inappropriate AP complement activation, as a result of either $\mathrm{fH}$ or membrane cofactor protein (MCP) dysfunction or gain-of-function mutations in $\mathrm{C} 3$ or factor $\mathrm{B}$ that render them more resistant to regulatory control (7). Complement has also been implicated in the pathogenesis of a number of common inflammatory and allergic disorders, including arthritis, ischemia-reperfusion injury, and asthma (7). Thus, anticomplement therapy represents a promising treatment strategy for a growing list of human conditions $(2,7,10)$.

Properdin (also referred to as $\mathrm{fP}$ ) is a plasma glycoprotein known as a positive regulator of AP complement activation. Discovered almost 50 years ago along with the AP, it was initially considered an initiator of this pathway $(11,12)$. Subsequent studies demonstrated that AP complement activation could be biochemically reconstituted in vitro in the absence of properdin, which led to the conclusion that properdin does not play an indispensable role in AP complement activation $(13,14)$. The widely accepted mode of action of properdin is that it binds and stabilizes surface-bound C3 convertase C3bBb, significantly extending its half-life $(15,16)$. Thus, initiation of the AP has been thought to be facilitated by properdin, but not dependent upon it. However, this traditional view has been challenged by recent studies showing that properdin can bind to target surface and initiate AP complement activation (17-22). Here, we examined the role of properdin in 2 models of AP complement activation in vivo. Our data revealed a critical role of properdin in mediating AP complement-mediated injury of host tissues and support therapeutic targeting of properdin as a potential treatment strategy for complement-dependent human diseases.

\section{Results}

Properdin deficiency rescues Crry ${ }^{-1-}$ mice from AP complement-mediated embryonic lethality. We have previously generated a properdin- 
null $\left(\mathrm{Cfp}^{-/}\right)$mouse by gene targeting (21). Using in vitro assays of diluted or neat serum, we found that LPS-induced AP complement activation was completely abrogated in the $C \mathrm{fp}^{-/-}$mouse serum, whereas zymosan-induced AP complement activation was only partially impaired (ref. 21 and data not shown). These data were suggestive of a varied requirement for properdin in AP complement activation, triggered by different microbial components. To determine the role of properdin in AP complement activation on susceptible host tissues, we used the Crry-/- mouse model $(23,24)$. Crry is a rodent-specific membrane complement regulator with DAF and MCP activities $(25,26)$. Gene deletion of Crry in mice caused embryonic lethality, such that no homozygous $\mathrm{Crry}^{-/-}$animals could be obtained from $\mathrm{Crry}^{+/-}$mating (23). However, embryonic lethality was preventable by deficiency of $\mathrm{C} 3$ or factor $\mathrm{B}-$ but not of $\mathrm{C} 4$ - in $\mathrm{Crry}^{+/-}$breeders, which suggests that AP complementmediated fetal injury was responsible for the lethal phenotype (24). Here, we crossed $\mathrm{Cfp}^{-/-}$mice with $\mathrm{Crry}^{+/-}$mice and generated $\mathrm{Cfp}^{-/-}$ $\mathrm{Crry}^{+/-}$mice as breeders. We then intercrossed $\mathrm{Cfp}^{-/-} \mathrm{Crry}^{+/-}$mice and examined 8 litters of progeny for their Crry genotype. Of 59 pups examined, we identified $14 \mathrm{Crry}^{+/+}, 28 \mathrm{Crry}^{+/-}$, and $17 \mathrm{Crry}^{-/-}$mice (24\%, $47 \%$, and $29 \%$, respectively). This outcome was not significantly different from the expected Mendelian distribution ratio $\left(P=0.79, \chi^{2}\right.$ test $)$ and mirrored the rescuing effects of $C 3$ and factor $B$ deficiency (23). Thus, properdin deletion protected $\mathrm{Crry}^{-/}$mouse fetus from AP complement-mediated lethal injury.

Properdin deficiency ameliorates disease in the $K / B \times N$ model of arthritis. The $\mathrm{K} / \mathrm{BxN}$ model of arthritis is a widely used murine model of inflammatory injury known to be mediated by AP complement (27). $\mathrm{K} / \mathrm{BxN}$ mice, generated by crossing the KRN TCR transgenic mouse on a C57BL/ 6 background with the NOD strain, develop an aggressive form of arthritis that is mediated by Abs against a common autoantigen, glucose-6-phosphate isomerase (GPI; ref. 28). Passive transfer of $\mathrm{K} / \mathrm{BxN}$ mouse serum or anti-GPI IgGs to naive mice caused similar destructive arthritis in the recipients (29). Using ankle thickening and clinical score as readouts, we first tested the serum transfer protocol for arthritis induction in naive WT mice and confirmed its dependency on C3 and factor B (Supplemental Figure 1; supplemental material available online with this article; doi:10.1172/ JCI41782DS1). We then evaluated disease induction in $\mathrm{Cfp}^{-/}$mice and their WT littermate controls and found that properdin deficiency significantly protected mice from arthritis development induced by serum transfer (Figure 1, A and B). Amelioration of arthritis in $C \mathrm{fp}^{-/-}$mice correlated with decreased tissue levels of the inflammatory cytokine IL-1 $\beta$ in the joint (Figure $1 \mathrm{C}$ ).

It is possible that adoptive transfer of $\mathrm{K} / \mathrm{BxN}$ mouse serum could partially reconstitute plasma properdin in $\mathrm{Cfp}^{-/-}$mice, which might have caused the full protective effect of properdin deficiency to be underestimated. Indeed, although no LPS-induced AP complement activity was observed in naive knockout mice, $C \mathrm{fp}^{-/-}$mice receiving $\mathrm{K} / \mathrm{BxN}$ mouse serum displayed transient and partial restoration of serum AP complement activity (Supplemental Figure 2A). To further evaluate this issue, we performed a comparative experiment in which purified total IgGs, instead of whole serum, from $\mathrm{K} / \mathrm{BxN}$ mice were used to induce disease. As expected, adoptive transfer of $\mathrm{K} / \mathrm{BxN}$ mouse IgGs did not confer AP complement activity to $\mathrm{Cfp}^{-/-}$mice (Supplemental Figure 2B).

Using this optimized protocol, we reevaluated arthritis development in WT and $\mathrm{Cfp}^{-/-}$mice and obtained similar results (Figure 1, $\mathrm{D}-\mathrm{F})$, with $\mathrm{Cf \textrm {p } ^ { - / - }}$ mice displaying significantly reduced ankle thickening and clinical scores (change in ankle thickness day 7 after IgG transfer, WT, $2.0 \pm 0.20 \mathrm{~mm}$; $\mathrm{Cfp}^{-/-}, 0.87 \pm 0.36 \mathrm{~mm}$; clinical index, WT, $3.6 \pm 0.44 ; C f p^{-/-}, 1.60 \pm 0.78 ; n=7$ [WT], $\left.11\left[\mathrm{Cfp}^{-/-}\right]\right)$. Histological analysis showed significantly reduced pathology scores (synovial hyperplasia, inflammatory infiltration, and pannus formation) and C3 staining (Figure 1, F and G), further corroborating the protective effect of properdin deficiency in this model of arthritis induction.

$B M$-derived cells are the major source of pathogenic properdin in $K / B x N$ arthritis. Human properdin has been reported to be synthesized by hepatocytes, various types of leukocytes, and endothelial cells (30-32). By Northern blot analysis, we previously detected Cfp mRNA in multiple mouse tissues, including the liver, spleen, and fat (21). To determine the source of properdin that contributes to arthritis development, we generated BM chimeras between WT and $C f p^{-/-}$mice. Real-time PCR analysis of Cfp mRNA in BM cells confirmed the successful reconstitution of donor BM in recipient mice (Figure 2A). Western blot analysis revealed BM cells to be the major source of plasma properdin, as its level in WT $\rightarrow \mathrm{Cfp}^{-/-}$ chimeras was similar to that of the WT $\rightarrow$ WT control, but was markedly reduced in $\mathrm{Cfp}^{-1-} \rightarrow \mathrm{WT}$ chimeras (Figure 2B). Likewise, LPS-induced AP complement activity in WT $\rightarrow \mathrm{Cfp}^{-/-}$chimeras was indistinguishable from that of WT mice, but was substantially reduced in $\mathrm{Cfp}^{-/-} \rightarrow \mathrm{WT}$ chimeras (Figure $2 \mathrm{C}$ ). We next tested the susceptibility of the chimeras to $\mathrm{K} / \mathrm{BxN}$ mouse serum-induced arthritis. WT $\rightarrow \mathrm{Cp}^{-1-}$ chimeras developed arthritis comparable in severity to that in the WT $\rightarrow$ WT control, whereas $\mathrm{Cfp}^{-/-} \rightarrow \mathrm{WT}$ chimeras developed a milder arthritis, marked by less ankle thickening and lower clinical scores (Figure 2, D and E). These data collectively suggest that $\mathrm{BM}$-derived cells are the major source of pathogenic properdin in $\mathrm{K} / \mathrm{BxN}$ arthritis. To determine the relative levels of properdin biosynthesis in different leukocyte populations, we performed real-time PCR analysis of purified splenic T $\left(\mathrm{CD}^{+}\right)$and $\mathrm{B}\left(\mathrm{CD} 19^{+}\right)$lymphocytes and macrophages/granulocytes $\left(\mathrm{CD} 11 \mathrm{~b}^{+}\right)$, and found the latter group had the most abundant properdin expression (Figure $2 \mathrm{~F}$ ).

Conditional gene targeting of properdin in myeloid lineage cells ameliorates $K / B x N$ arthritis. To further define the cellular source of properdin involved in AP complement-mediated injury, we used gene targeting with the Cre/lox system to generate tissue-specific Cfp knockout mice. Our targeting strategy was to flank exons 3-5 of the Cfp gene with 2 loxP sites (Supplemental Figure 3, A-C). This was the same targeting strategy that previously led us to the serendipitous creation of $C \mathrm{fp}^{-/-}$mice by NEO insertion (21). We used a 3'probe for Southern blot analysis to screen for positive ES cell clones (21), followed by second-round screening by PCR and restriction enzyme digestion to confirm correct $5^{\prime}$ recombination (Supplemental Figure 3, C and D). Of the ES cell clones identified by Southern blot, the majority did not have correct $5^{\prime}$ targeting and thus lacked the $5^{\prime}$ loxP site, as we have found previously (21). However, 1 of 7 clones was confirmed to have undergone correct recombination at both $3^{\prime}$ and $5^{\prime}$ ends, resulting in 2 loxP sites flanking exons 3-5 as intended (Supplemental Figure 3, $\mathrm{C}$ and D). This ES cell clone was used to generate chimeric mice that subsequently transmitted the mutation through germline (data not shown). Because we found previously that NEO insertion completely inactivated $C f p$, but the phenotype was reversed by NEO excision (21), we crossed the Cfp-floxed mouse with the FLPe transgenic mouse and removed the NEO gene cassette (data not shown). This step allowed us to generate a mouse with a functional but floxed Cfp gene (Cfpl/fl; Supplemental Figure 4). 

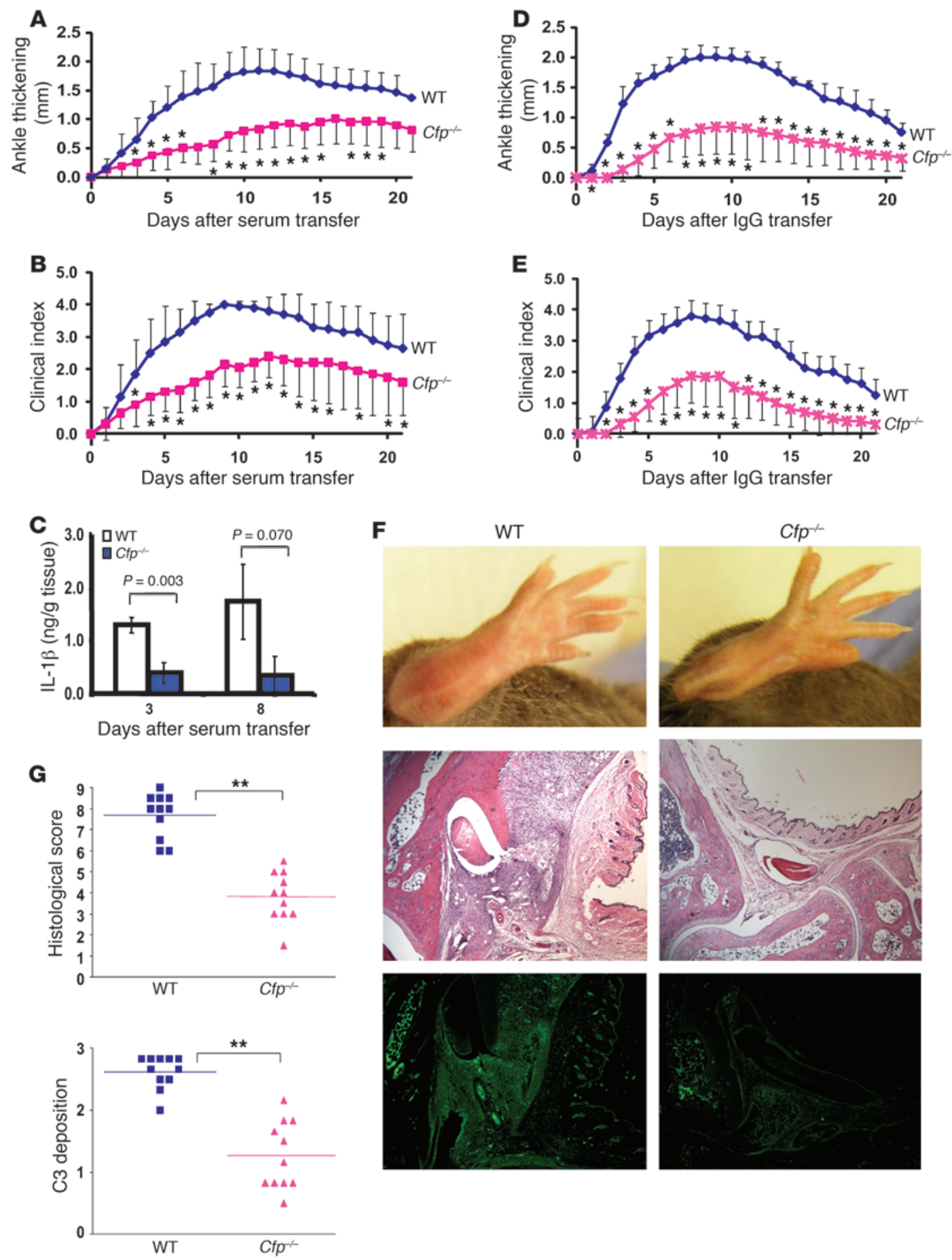

Figure 1

Properdin deficiency ameliorates $\mathrm{K} / \mathrm{BxN}$ arthritis. Arthritis was induced in $\mathrm{Cfp}^{-/-}$mice and their WT littermate controls by i.p. injection of $\mathrm{K} / \mathrm{BxN}$ mouse serum (A and $\mathbf{B} ; n=10$ per group) or $\operatorname{lgG}\left(\mathbf{D}\right.$ and $\mathbf{E} ; n=7[\mathrm{WT}], 11\left[\mathrm{Cfp}^{-/-}\right]$) on days 0 and 2 . Ankle thickening $(\mathbf{A}$ and $\mathbf{D})$ and clinical index ( $B$ and $\mathbf{E}$ ) were recorded daily. Ankle thickness was measured in millimeters by a caliper and presented as change from day 0 . Clinical index was determined as follows: 1 point for each inflamed and swollen paw; 0.5 points for each paw with mild swelling/redness or a few affected digits. ${ }^{*} P<0.05$ versus WT, nonparametric Wilcoxon/Kruskal-Wallis test. (C) IL-1 $\beta$ levels in tissue homogenates of ankles $(n=3$ per group) at days 3 and 8 after K/BxN serum transfer. $P$ values were determined by Student's $t$ test. $(\mathbf{F})$ Representative images of arthritis in WT and Cfp ${ }^{-/-}$mice at day 7 after K/BxN IgG transfer showing gross anatomy of hind limbs (top), joint histology with H\&E staining (middle), and C3 staining (bottom). Original magnification, $\times 40$. (G) Semiquantitative scoring of histopathology and C3 deposition in the joints of WT and Cfp $p^{-1-}$ mice at day 7 after $\mathrm{K} / \mathrm{BxN}$ IgG transfer. A total of 11 hind limb joints from 6 mice in each group of mice was analyzed; horizontal bars denote mean. ${ }^{* \star} P<0.001$, Student's $t$ test. 

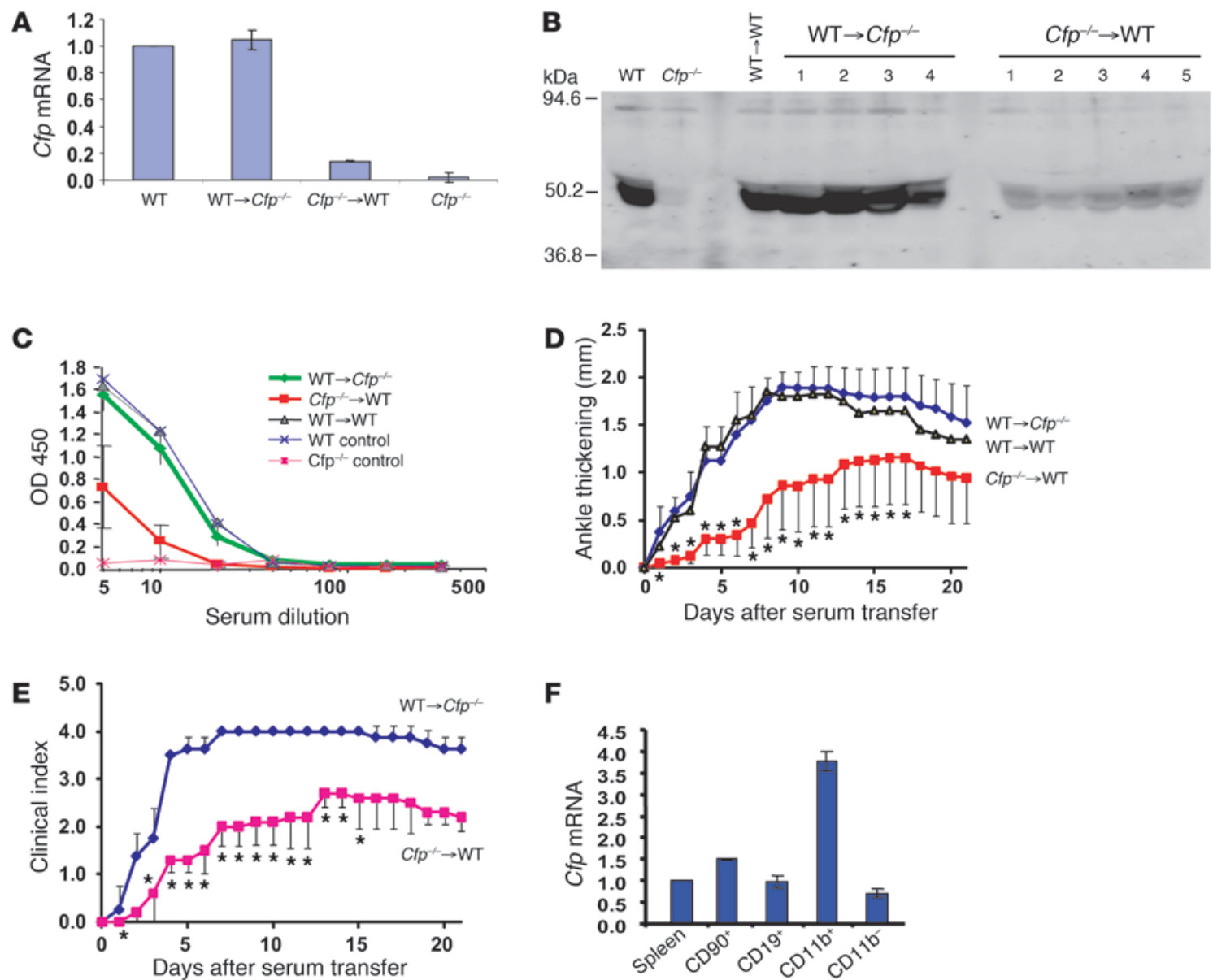

\section{Figure 2}

BM-derived cells are the major source of pathogenic properdin in K/BxN arthritis. (A) Real-time PCR analysis of Cfp mRNA levels in BM cells of chimeric mice. Gapdh was used as an internal control, and values were normalized to those of WT mice. (B) Western blot analysis of properdin in the plasma ( $2 \mu \mathrm{l} /$ lane) of chimeric mice. Numbers refer to individual mice. WT and $\mathrm{Cfp}^{-/}$mouse plasmas were used as controls. Rabbit antimouse properdin serum was used as the primary Ab. (C) ELISA plate assay of LPS-induced AP complement activity in sera of chimeric mice. (D) K/BxN serum-induced arthritis in chimeric mice, as assessed by changes in ankle thickness (relative to day 0 ). ${ }^{*} P<0.05$ versus WT $\rightarrow C f p^{-/-}$, nonparametric Wilcoxon/Kruskal-Wallis test. (E) K/BxN serum-induced arthritis in chimeric mice, as assessed by clinical score. ${ }^{*} P<0.05$ versus WT $\rightarrow \mathrm{Cfp}^{-/-}$, nonparametric Wilcoxon/Kruskal-Wallis test. (F) Real-time PCR analysis of Cfp mRNA levels in splenic T (CD90+) and B (CD19+) lymphocytes and granulocytes/macrophages $\left(\mathrm{CD} 11 \mathrm{~b}^{+}\right)$. All 3 types of cells were positively selected from splenocytes (pooled from 8 WT mice) by column purification. CD11 b- cells were the leftover fraction from CD11 belection. Gapdh was used as an internal control, and values were normalized to that of total splenocytes (Spleen). Values represent mean \pm SD of triplicate PCR assays.

To test Cre-mediated properdin inactivation, we first crossed the $C \mathrm{fp}^{f l / f l}$ mouse with the Ella-Cre transgenic mouse, which is capable of causing ubiquitous deletion of a floxed target gene (33). We confirmed that $C f p^{f l / f l}$ Ella-Cre- mice had intact exons 3-5 in their $C f p$ gene structure, whereas $C f p^{f / f l}$ Ella-Cre ${ }^{+}$mice had these exons successfully deleted (Supplemental Figure 4, A-C). Furthermore, no LPS-induced AP complement activity was detected in the sera of $C \mathrm{fp}^{f / f l} \mathrm{Ella}-\mathrm{Cr} \mathrm{e}^{+}$mice (Supplemental Figure 4D). These results thus validated the Cre/lox system for efficient properdin deletion in this mouse model.

We next crossed the $C f p^{f l / f l}$ mouse with the lysozyme-Cre (LysCre) transgenic mouse, which expresses the Cre recombinase specifically in myeloid lineage cells (34). $C f p^{f l / f l} \mathrm{Lys}^{-\mathrm{Cre}^{+}}$mice, but not $C f p^{f / f l}$ Lys-Cre ${ }^{-}$mice, had diminished $C f p$ mRNA expression in their splenic CD11b+ cells (Figure 3A). However, no reduction in
Cfp mRNA was observed in splenic B cells of Cfp $\mathrm{p}^{f / f l} \mathrm{Lys}-\mathrm{Cre} \mathrm{e}^{+}$mice, which confirmed that Cre-mediated properdin deletion in these mice was specific to myeloid lineage cells. Importantly, Western blot analysis revealed a dramatic reduction of plasma properdin level in $C f p^{f l / f l}$ Lys-Cre $e^{+}$mice compared with WT or Cfpt/fll Lys-Cre ${ }^{-}$ littermate controls (Figure 3B). Likewise, we detected a significant decrease in LPS-induced AP complement activity in the sera of $C f \mathrm{p}^{f / f l}$ Lys-Cre ${ }^{+}$mice (Figure 3C). Finally, when tested in the K/BxN model of arthritis with adoptive total IgG transfer, $C f^{f l / f l}$ Lys-Cre ${ }^{+}$ mice developed significantly attenuated disease compared with $C \mathrm{fp}^{f / f} \mathrm{fl}_{\text {Lys-Cre }}$ littermate controls (Figure 3, D and E). Collectively, these data suggest that plasma properdin is largely derived from myeloid lineage cells and that properdin synthesized by these cells plays a critical role in AP complement-mediated injury in vivo. To ascertain whether properdin - in systemic circulation or released 
A

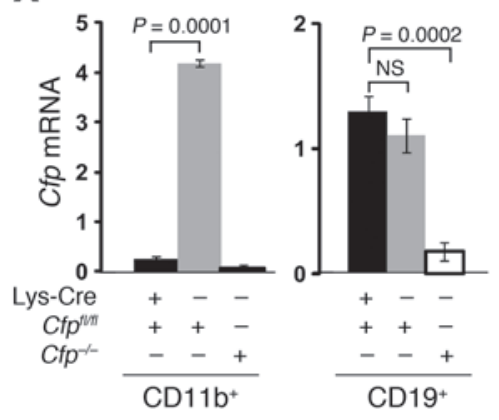

D

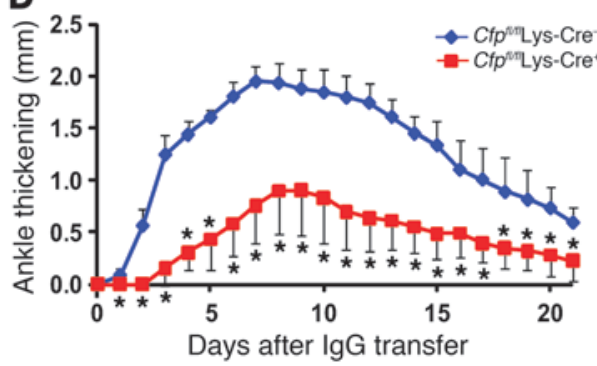

$\mathbf{F}$

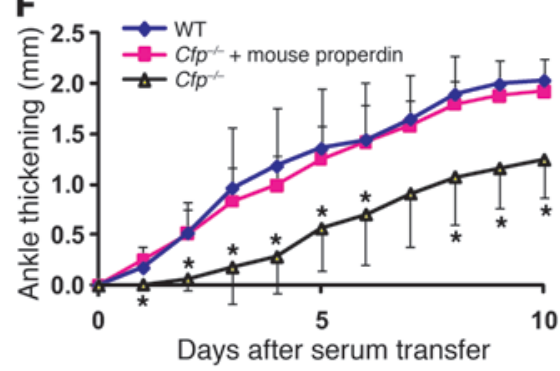

B

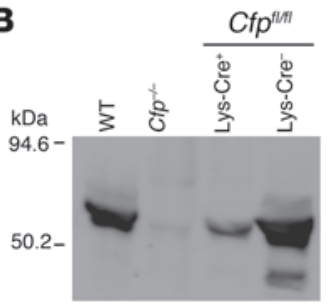

C

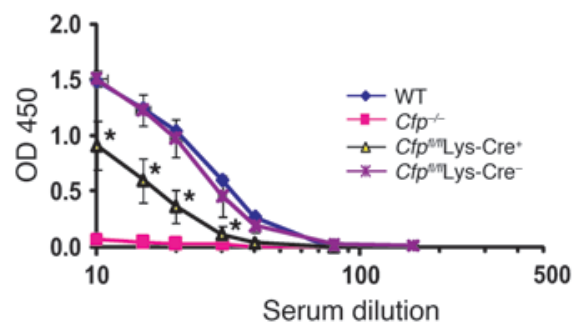

E

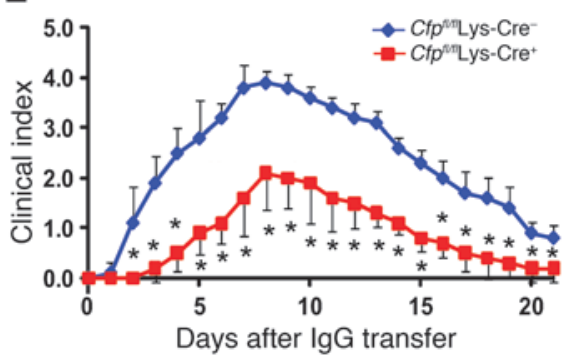

G

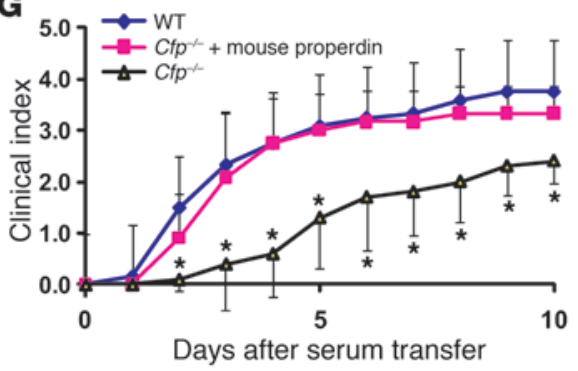

Figure 3

Myeloid lineage cell-derived properdin plays a critical role in AP complement-mediated injury. (A) Real-time PCR analysis of Cfp mRNA

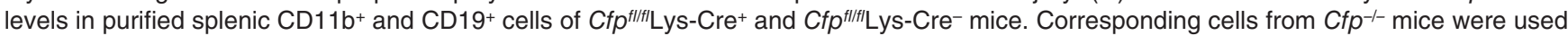
as negative controls. $P$ values were determined by Student's $t$ test. (B) Western blot analysis of plasma properdin levels in $C f p^{f / / f} L y s-C r e^{+}$and $\mathrm{Cfp}^{f / f / \mathrm{L}} \mathrm{Lys}-\mathrm{Cre} \mathrm{e}^{-}$mice. WT and $\mathrm{Cfp}^{-/-}$mouse sera were used as controls. Total protein from each serum sample (12.5 $\left.\mu \mathrm{l}\right)$ was precipitated by $5 \%$ PEG before SDS-PAGE separation, and rabbit anti-mouse properdin serum was used as the primary Ab. (C) LPS-induced AP complement

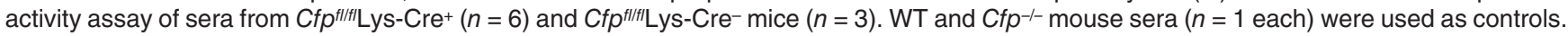
(D and E) K/BxN mouse IgG-induced arthritis, as assessed by ankle thickening relative to day 0 (D) and clinical index (E), in Cfp fl/fl/Lys-Cre+ and $\mathrm{Cfp}^{f / / f /} \mathrm{Lys}-\mathrm{Cre}^{-}$mice $\left(n=5\right.$ per group). ( $\mathbf{F}$ and $\mathbf{G}$ ) Restoration of arthritis sensitivity to $\mathrm{Cfp}^{-/-}$mice by exogenous mouse properdin administration $(100 \mu \mathrm{g}$, i.p., 6 hours prior to $\mathrm{K} / \mathrm{BxN}$ serum transfer). Arthritis was assessed by ankle thickening relative to day $0(\mathbf{F})$ and by clinical index $(\mathbf{G})$ in

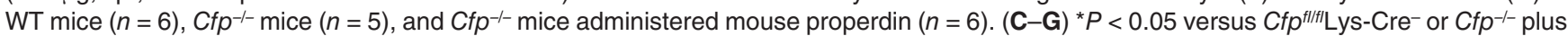
mouse properdin, nonparametric Wilcoxon/Kruskal-Wallis test.

locally by infiltrating leukocytes within the joints - is responsible for AP complement-mediated injury, we treated $\mathrm{Cfp}^{-1-}$ mice with purified mouse properdin (see below for mouse properdin purification and characterization). Replacement of exogenous properdin reconstituted serum AP complement activity to $\mathrm{Cfp}^{-/-}$mice (data not shown) and fully restored their sensitivity to $\mathrm{K} / \mathrm{BxN}$ seruminduced arthritis (Figure 3, F and G). This result thus suggested that systemic properdin from the circulation is responsible for causing AP complement-mediated injury in $\mathrm{K} / \mathrm{BxN}$ arthritis.

$A b$ neutralization of properdin protects WT mice from $K / B \times N$ arthritis. The finding that systemic properdin was pathogenic in $\mathrm{K} / \mathrm{BxN}$ arthritis suggested that properdin may be a feasible therapeutic target in AP complement-mediated diseases. As a proof of concept, we produced a function-blocking polyclonal anti-mouse properdin
$\mathrm{Ab}$ to test in WT mice subjected to arthritis induction. We purified properdin from pooled mouse plasma by fast protein liquid chromatography (FPLC) using restoration of LPS-dependent AP complement activity to $\mathrm{Cfp}^{-/-}$mouse serum as a bioassay. The most active fractions from the last FPLC step were confirmed to contain a protein of near homogeneity with the expected molecular weight of mouse properdin (Figure 4A). These fractions were pooled and used for immunization of a rabbit to produce a polyclonal $\mathrm{Ab}$ that reacted with mouse plasma properdin with high specificity (Figure 4B). We then purified the total IgG fractions from nonimmune and anti-properdin rabbit sera and prepared the corresponding $\mathrm{F}\left(\mathrm{ab}^{\prime}\right)_{2}$ fragments by pepsin digestion followed by FPLC purification. Anti-mouse properdin $\mathrm{F}\left(\mathrm{ab}^{\prime}\right)_{2}$, but not the nonimmune control $\mathrm{F}\left(\mathrm{ab}^{\prime}\right)_{2}$, completely blocked LPS-induced AP complement 

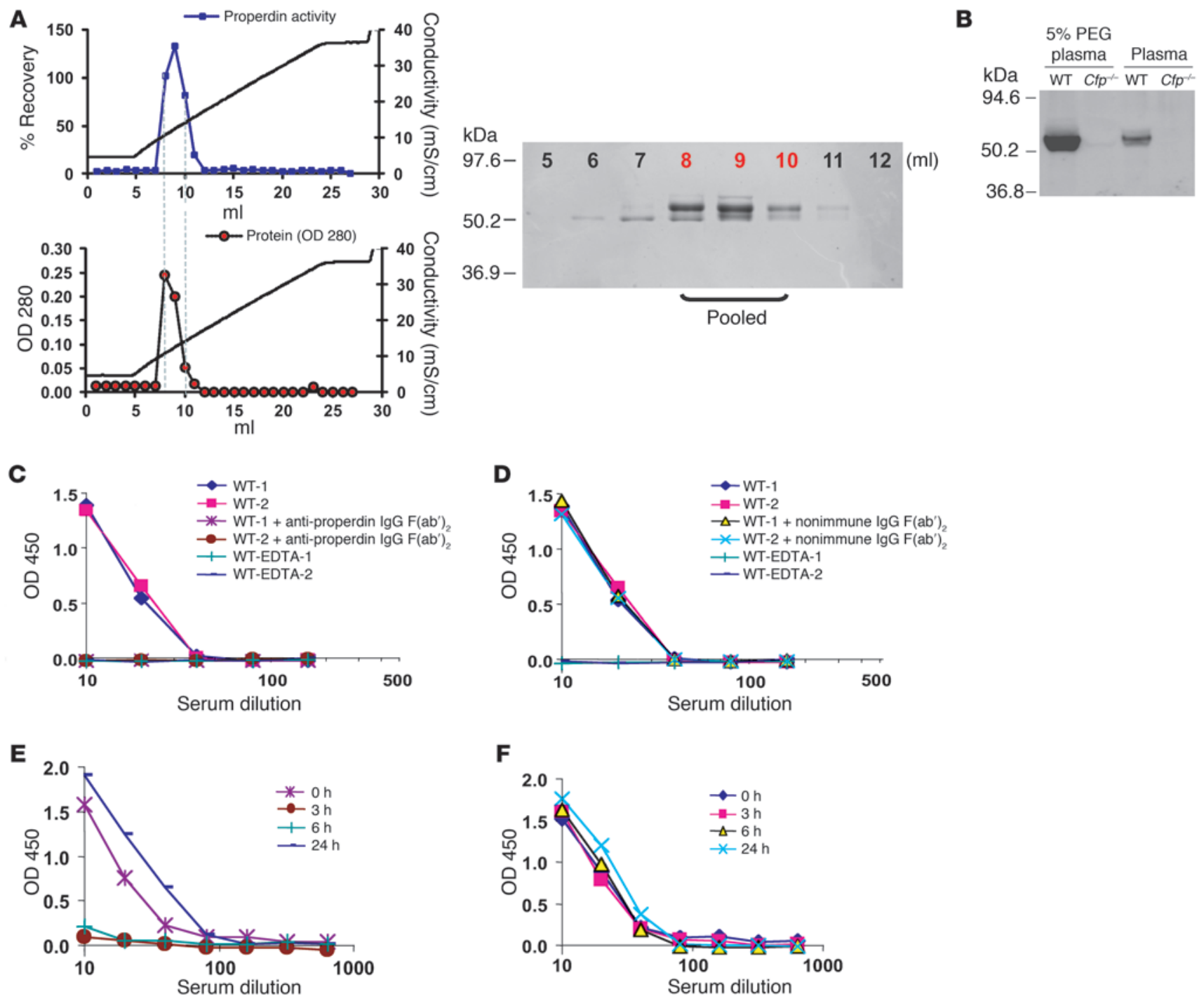

Figure 4

Purification of mouse properdin and generation of a function-blocking anti-mouse properdin Ab. (A) Elution of properdin from the Mono $\mathrm{S}$ column of FPLC with a salt gradient (20-300 mM in 20 minutes). Protein concentration in the FPLC fractions was monitored by OD280, and its activity was determined by LPS-induced AP complement activation assay using $\mathrm{Cfp}^{-/-}$mouse serum. Data are presented as percent recovery of $\mathrm{AP}$ complement activity in $\mathrm{Cfp}^{-/-}$mouse serum (normalized to WT mouse serum). Fractions 5-12 (10 $\mu$ l each) were analyzed for purity by separation on a $10 \%$ SDS-PAGE gel under reducing condition and staining with Coomassie blue. The most active fractions from the last FPLC step (fractions 8-10; red font), confirmed to contain a protein of near homogeneity with the expected molecular weight of mouse properdin, were pooled. (B) Western blot assay showing reactivity of a rabbit polyclonal anti-mouse properdin Ab with plasma properdin in WT mice. No signal was detected in $\mathrm{Cfp}^{-/-}$mouse plasma. Plasma samples were either analyzed directly (2 $\mu \mathrm{l}$ ) or precipitated with $5 \%$ PEG (from $\left.12.5 \mu \mathrm{l}\right)$. (C and D) Ab neutralization of plasma properdin in vitro. Rabbit anti-mouse properdin IgG $F\left(a b^{\prime}\right)_{2}(\mathbf{C})$, but not nonimmune rabbit lgG $F\left(a b^{\prime}\right)_{2}(\mathbf{D})$, blocked LPS-induced AP complement activation in WT mouse serum. EDTA sera were used as negative controls. (E and F) Ab neutralization of plasma properdin in vivo. WT mice were injected with rabbit anti-mouse properdin IgG $F\left(a b^{\prime}\right)_{2}(E)$ or nonimmune rabbit IgG $F\left(a b^{\prime}\right)_{2}(F)(2 \mathrm{mg} / \mathrm{mouse}$ i.p.). Serum samples were collected at the indicated times and assayed for LPS-induced AP complement activity.

activity in WT mouse serum (Figure 4, C and D). To test the efficacy of anti-properdin $\mathrm{F}\left(\mathrm{ab}^{\prime}\right)_{2}$ in vivo, we administered a bolus dose ( $2 \mathrm{mg} /$ mouse) to WT mice and assessed serum AP complement activity at various time points. Whereas nonimmune $\mathrm{F}\left(\mathrm{ab}^{\prime}\right)_{2}$ had no effect, anti-mouse properdin $\mathrm{F}\left(\mathrm{ab}^{\prime}\right)_{2}$ suppressed serum $\mathrm{AP}$ complement activity for at least 6 hours (Figure 4, E and F). AP complement activity in the treated mice returned to normal levels by 24 hours (Figure 4E). Importantly, as with Cfp deletion, treatment of WT mice with anti-properdin $\mathrm{F}\left(\mathrm{ab}^{\prime}\right)_{2}$ significantly ameliorated $\mathrm{K} / \mathrm{BxN}$ mouse serum-induced arthritis (Figure 5). These results demonstrated that it is feasible to ameliorate local complement injury by systemically targeting properdin.

\section{Discussion}

Recently, there has been a resurgence of interest in the plasma protein properdin in complement activation $(16,35)$. Although 


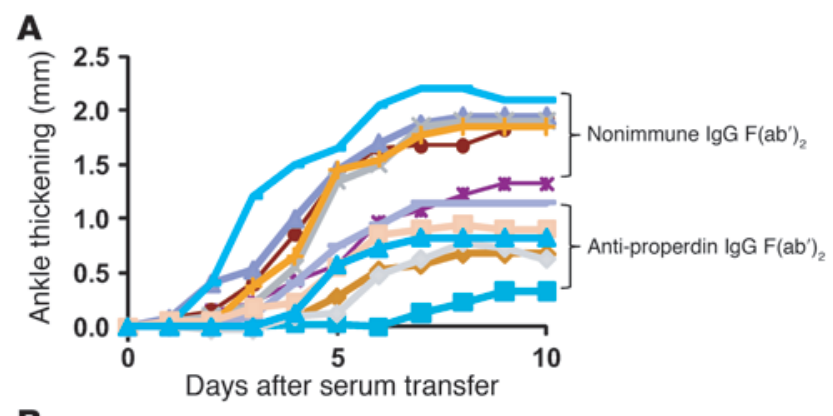

\section{B}

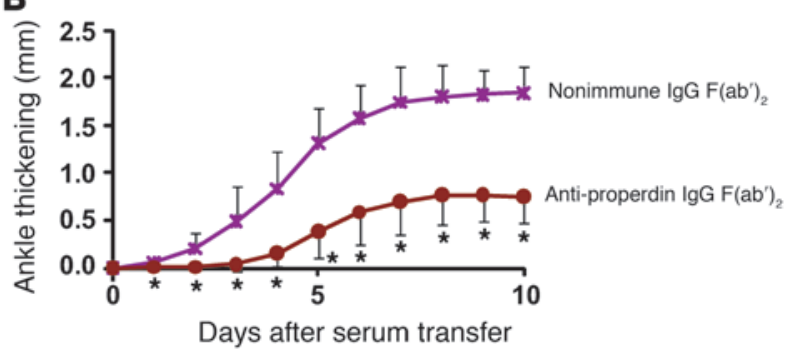

C

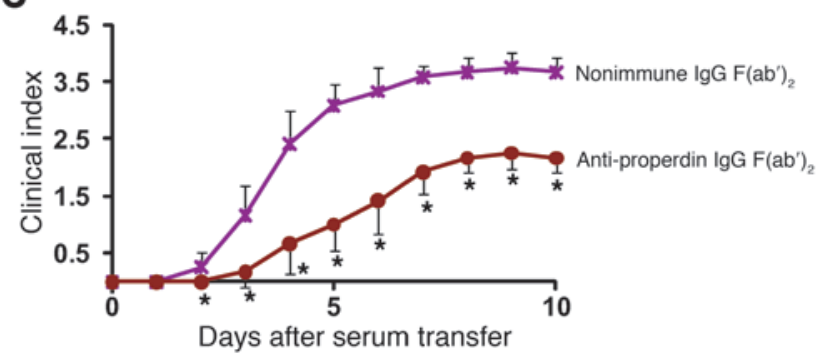

Figure 5

Ab neutralization of properdin protects WT mice from K/BxN arthritis. (A) Ankle thickening (relative to day 0 ) of each individual mouse treated with anti-mouse properdin IgG $\mathrm{F}\left(a b^{\prime}\right)_{2}$ or nonimmune rabbit IgG $\mathrm{F}\left(a b^{\prime}\right)_{2}$ ( $n=6$ per group). (B and C) Average ankle thickening (B) and clinical index (C) of mice in $\mathbf{A}$. Mice were injected with anti-properdin or nonimmune rabbit IgG $F\left(a b^{\prime}\right)_{2}(2 \mathrm{mg} /$ mouse i.p.) 30 minutes before each $\mathrm{K} / \mathrm{BxN}$ serum transfer (i.e., on days 0 and 2). ${ }^{\star} P<0.05$ versus nonimmune rabbit IgG $F\left(a b^{\prime}\right)_{2}$, nonparametric Wilcoxon/Kruskal-Wallis test.

considered a key component for AP complement activation in the early days after its discovery (11), properdin has since been largely regarded as a facilitating but dispensable regulator of the AP (16). Properdin exists in the form of multimers in the plasma and is believed to promote AP complement activation by binding and stabilizing the newly formed AP C3 convertase C $3 \mathrm{bBb}$, extending its half-life from 1-2 minutes to more than 18 minutes (15). Several recent studies have prompted reexamination of the textbook view of the mechanism of action of properdin. Hourcade and colleagues have demonstrated that, in addition to working as a stabilizer, surface-immobilized properdin could serve as an anchor for new $\mathrm{C} 3 \mathrm{bBb}$ assembly to initiate AP complement activation (17, $18,20,35)$. We have previously shown that purified properdin was able to bind to LPS, and the LPS/properdin complex triggered avid AP complement activation in properdin-deficient serum, which otherwise lacked detectible LPS-induced AP complement activity (21). These findings suggested that properdin could function as a pattern recognition molecule to initiate the AP and play an indispensable role in LPS-triggered AP complement activation. On the other hand, it appears that not all microbial activators of the AP complement require properdin. For example, unlike LPS, zymosan was capable of triggering robust AP complement activation in $C \mathrm{fp}^{-/-}$mouse serum (21).

The major finding of the present study was a critical role for properdin in AP complement-mediated autologous tissue injury. Thus, properdin represents an attractive therapeutic target for human diseases caused by inappropriate AP complement activation. The critical role of properdin in AP complement activation on self-tissues may reflect the fact that host cells are usually well protected by multiple complement inhibitors, and a positive regulator like properdin is therefore necessary to tip the balance and overcome the inhibitory mechanisms. It should be noted that although our data indicated a critical role of properdin in this process, they could not differentiate whether properdin acts as an initiator by binding to susceptible host cells or as a stabilizer of C3bBb formed on the cell surface. A second conclusion of our study was that systemic properdin derived from myeloid lineage cells appeared to be responsible for arthritis pathogenesis. It has been postulated that infiltrating leukocytes may release properdin locally at the site of inflammation to trigger AP complement activation and exacerbate tissue injury $(20,36)$. Although our results do not discount a role of freshly released properdin acting locally, the finding that systemic reconstitution of $\mathrm{Cfp}^{-/-}$mice with exogenous properdin restored $\mathrm{K} / \mathrm{BxN}$ arthritis sensitivity suggests that circulating properdin is capable of causing local injury in the joint.

An important consideration in developing anticomplement therapies is the balance between the beneficial effect of preventing tissue injury and the detriment of possibly compromising host defense. Properdin deficiency in humans has been reported to be associated with meningococcal infection in some individuals, possibly in the context of low $\mathrm{Ab}$ (37) and/or serum mannose-binding lectin levels (38). Nevertheless, it could be argued that anti-properdin therapy offers several advantages over those targeting other complement proteins, such as $\mathrm{C} 3, \mathrm{fB}, \mathrm{fD}$, and $\mathrm{C} 5$ (10). First, the finding that not all microbial AP complement activators required properdin (e.g., LPS, but not zymosan, depended on properdin to trigger the AP complement of the mouse) suggests that blocking properdin function may affect AP complement activity against some pathogens, but not all. Second, there is some evidence that properdin does not play a critical role in the AP amplification loop of the classical pathways (ref. 21 and our unpublished observations). Should further studies confirm the lack of involvement of properdin in the classical and lectin pathways, then therapeutic targeting of properdin will not compromise host defense mediated by these pathways. Third, unlike eculizumab, a C5-blocking $\mathrm{mAb}$, pharmacological targeting of properdin would block adverse effects of AP complement mediated by C3a/C3b as well as C5a/ MAC. Finally, the relatively low plasma level of properdin (5-15 $\mu \mathrm{g} / \mathrm{ml}$; ref. 36) makes it more amenable for therapeutic manipulation. In principle, the potential risk of meningococcal infection posed by antiproperdin therapy could be mitigated by prophylactic vaccination of patients, a strategy that has been successfully used in the treatment of PNH patients with eculizumab $(8,9)$.

In summary, we have revealed a critical role of properdin in AP complement-mediated injury. We have also provided proof of principle for systemically targeting properdin as a way to ameliorate AP complement-mediated end-organ damage. Collectively, our data support the development of antiproperdin therapy for arthritis and other genetically predisposed human diseases, such as aHUS and 
AMD, that have recently been linked to dysregulated AP complement and for which no treatment is currently available.

\section{Methods}

Mice and conditional gene targeting experiment. WT C57BL/6, FLPe transgenic, Ella-Cre transgenic, Lys-Cre transgenic, and NOD mice were obtained from the Jackson Laboratory. KRN TCR transgenic mice were provided by D. Mathis (Harvard Medical School, Boston, Massachusetts, USA). $\mathrm{Crry}^{+/-}$mice were provided by H. Molina (Washington University, St. Louis, Missouri, USA). $C \mathrm{fp}^{-/-}$mice were produced by NEO insertion through gene targeting, as previously described (21). To generate a conditional Cfp knockout mouse, we used the same targeting strategy and screened ES cells for correct targeting at both $3^{\prime}$ and $5^{\prime}$ ends of the homologous sequence (Supplemental Figure 3, A and B). ES cells were first screened by Southern blot for correct $3^{\prime}$ targeting, as described previously (21). Positive clones were then screened by PCR and restriction enzyme digestion (Supplemental Figure 3, C and D). Briefly, PCR was performed using genomic DNAs from the positive ES cell clones and the following primers that span the 5' loxP site: 5'-GTAAAGATGATAGGCTTGAAGAT-3' and 5'-CCTAGCAGTACTGCTCATGTG-3' (904 bp for WT and 944 bp for the targeted clones). The amplified PCR fragment was then subjected to EcoRV digestion to identify positive clones containing an embedded EcoRV site adjacent to the loxP sequence. A single positive ES cell clone, A3, was identified by this 2-round screening procedure (Supplemental Figure 3, C and D) and was used for chimeric mouse production, as previously described (21). High percentage male chimeras were mated with C57BL/6 mice to achieve germline transmission. Progeny with a targeted C $f p$ gene were identified by PCR for the presence of Neo in their genomic DNA using NEO-specific primer 5'-GGGTGGGATTAGATAAATGCC-3' and properdin-specific primer 5'-CAAGGTACGGCTTTGTTACACA-3' ( $\approx 700$-bp product expected). Female NEO-positive mice were crossed with FLPe transgenic mice to delete the NEO gene cassette (39) and produce Cfp $\mathrm{fl}^{\mathrm{f} / \mathrm{fl}}$ mice. To detect the FLPe transgene by PCR, the following primers were used: 5'-CACTGATATTGTAAGTAGTTTGC-3' and 5'-CTAGTGCGAAGTAGTGATCAGG-3' (725-bp product expected). To detect loxP sequence as a marker of targeted gene allele after NEO excision, the following 2 primers were used: $5^{\prime}$-ATAACTTCGTATAATGTATGCTATACGAAGTTAT- $3^{\prime}$ and 5'-CAAGGTACGGCTTTGTTACACA-3' ( $\approx 400$-bp product).

To test the efficiency of Cre/lox-mediated properdin inactivation, $C f p^{f / f l}$ mice were crossed with the Ella-Cre transgenic mouse, which expresses Cre in oocytes and is often used as a universal deleter of floxed genes (40). The Cre transgene was screened by PCR using 5 '-ATTCTCCCACCGTCAGTACG-3' and $5^{\prime}$-CGTTTTCTGAGCATACCTGGA- $3^{\prime}$ as primers $(\approx 500$-bp product expected; ref. 41). Exon 3-5 deletion in $\mathrm{Cfp}^{f / f l} \mathrm{Ella}^{\mathrm{l}} \mathrm{Cre}^{+}$mice was detected by PCR of tail DNA using the following primers: 5'-CAGAGGAAAATGAAGGGAAATG-3' and 5'-CAAGGTACGGCTTTGTTACACA-3' (1,968-bp for WT and $\approx 400$-bp for knockout; Supplemental Figure 4, A-C). To generate mice deficient of properdin in myeloid lineage cells, $C \mathrm{fp}^{l / f l}$ mice were crossed with the Lys-Cre transgenic mouse (34). Progeny was screened by PCR for the Cre transgene, and positive mice were examined for properdin inactivation in their $\mathrm{CD} 11 \mathrm{~b}^{+}$or $\mathrm{CD} 19^{+}$splenic cells (Figure $3 \mathrm{~A}$ ). Unless otherwise specified, all mice used had a mixed C57BL/6-129J background, and WT littermates were used as controls. All animal experiments were approved by the Institutional Animal Care and Use Committee of the University of Pennsylvania.

Arthritis induction. $\mathrm{K} / \mathrm{BxN}$ mice were generated by breeding male KRN mice with female NOD mice. $\mathrm{K} / \mathrm{BxN}$ mouse serum was collected from animals between 8 and 10 weeks of age. Prior to arthritis induction, the titer of each batch of collected serum was analyzed, and optimal serum dose was calculated as previously described $(28,42,43)$. For induction of arthritis,
$150-200 \mu \mathrm{l}$ of pooled serum from $\mathrm{K} / \mathrm{BxN}$ mice was injected i.p. at days 0 and 2 to 6 -week-old recipient mice. In an alternative protocol, arthritis was induced in recipient mice by passive transfer of purified total IgGs from $\mathrm{K} / \mathrm{BxN}$ mice (2 mg/mouse i.p.). Ankle thickening was measured by a caliper (Mitutoyo), and clinical scores were recorded using previously published criteria $(29,44)$. In some experiments, $C f p^{-/-}$mice received freshly purified (i.e., not subjected to freezing and thawing) mouse properdin $(100 \mu \mathrm{g} /$ mouse i.p.) 6 hours before $\mathrm{K} / \mathrm{BxN}$ serum treatment, and WT mice received anti-properdin $\operatorname{IgG~} \mathrm{F}\left(\mathrm{ab}^{\prime}\right)_{2}$ fragments $(2 \mathrm{mg} /$ mouse i.p.) 30 minutes before each $\mathrm{K} / \mathrm{BxN}$ serum treatment.

Histology and cytokine assays. H\&E staining of the joints was performed as described previously (45). In brief, hind limb joints were removed immediately after mice were euthanized. They were fixed in $4 \%$ paraformaldehyde overnight and then decalcified in 6\% nitric acid for 8 hours. This treatment (fixation and decalcification) was repeated once, and the tissue was processed for paraffin embedding. Paraffin blocks were sectioned at $5 \mu \mathrm{m}$ and processed for $\mathrm{H} \& \mathrm{E}$ staining. Histopathological grading was performed as previously described $(46,47)$ using the following morphological parameters of synovitis: (a) hyperplasia/enlargement of synovial lining layer; (b) degree of inflammatory infiltration; and (c) activation of synovial stroma/ pannus formation. Each parameter was graded as 0 , absent; 1 , slight; 2 , moderate; and 3 , strong; totals for all 3 parameters ranged from 0 to 9 . C3 deposition was determined by staining paraffin-embedded slides with FITC-conjugated polyclonal goat anti-mouse C3 Abs (MP biomedicals). Scoring of C3 staining intensity of the synovium was performed using a 3 -point scoring system as previously described (48). Each slide was scored by 2 trained individuals. For each joint sample, 3 separate slides were read, and the values were averaged.

To determine intraarticular cytokine levels, hind footpads from arthritic mice (days 0,3 , and 8 after $\mathrm{K} / \mathrm{BxN}$ serum transfer) were cut at the borderline of fur growth and frozen in liquid nitrogen. Tissues were homogenized in $75 \mathrm{mg} / \mathrm{ml}$ PBS, and supernatant was prepared for ELISA determination of IL-1 $\beta$, TNF- $\alpha$, and IL-6 levels. All ELISA kits were from BD Biosciences, and assays were performed according to the manufacturer's instructions.

$B M$ chimera experiment. BM chimeric mice were generated as previously described $(49,50)$. Briefly, BM cells from femurs of 8-week-old male donor mice were flushed out with culture medium using a $3-\mathrm{ml}$ syringe (BD Biosciences). $2 \times 10^{6}$ cells were injected into the tail vein of each lethally irradiated $(950 \mathrm{R}=6.3 \mathrm{~Gy})$ syngeneic recipient mouse. We generated 2 main types of chimeras, WT $\rightarrow \mathrm{Cfp}^{-/-}(n=4)$ and $\mathrm{Cfp}^{-1-} \rightarrow \mathrm{WT}(n=5)$. A third type, $\mathrm{WT} \rightarrow \mathrm{WT}(n=1)$, was also generated as a control. Chimeric mice were used for experiments 3 months after BM transfer.

Purification of mouse properdin. Mouse properdin was purified from pooled plasma (Innovative Research) using a modified protocol based on human properdin purification (51). Stock solutions of polyethylene glycol (PEG; $50 \%$ ) and PMSF (100 mM) were mixed with $50 \mathrm{ml}$ mouse EDTA-plasma to give a final concentration of $5 \%$ PEG and $0.5 \mathrm{mM}$ PMSF. After centrifugation at $8,000 \mathrm{~g}$ for 30 minutes, the supernatant was removed, and the pellet was resuspended in $20 \mathrm{ml}$ buffer A $(20 \mathrm{mM}$ phosphate and $20 \mathrm{mM} \mathrm{NaCl}$, $\mathrm{pH}$ 7.2) and used as a starting material for FPLC column chromatography. The sample was first filtered $(0.22 \mu \mathrm{m})$, and $10 \mathrm{ml}$ each was applied onto a HiTrap Q 5-ml column (GE Healthcare) equilibrated in buffer A. The column flow-through (containing properdin) was subsequently applied onto a Mono Q HR 5/5 column (GE Healthcare) equilibrated with buffer A. The flow-through fraction was again collected and applied onto a cation exchange Mono S HR 5/5 column (GE Healthcare) equilibrated with buffer $\mathrm{A}$. The Mono $\mathrm{S}$ column was then washed with a salt gradient $(0-300$ $\mathrm{mM}$ over 20 minutes $)$ in buffer B $(20 \mathrm{mM}$ phosphate and $500 \mathrm{mM} \mathrm{NaCl}$, $\mathrm{pH}$ 7.2), and 1-ml fractions were collected. Properdin-containing fractions were identified by SDS-PAGE and a functional assay of LPS-dependent AP 
complement activity using $\mathrm{Cfp}^{-/-}$serum as a source of other complement components. Briefly, $\mathrm{Cfp}^{-/-}$mouse serum (1:15 dilution) was reconstituted with FPLC fractions (1:500 dilution) and tested for AP complement activity on LPS-coated ELISA plates, as described previously (21). To estimate the properdin concentration in the peak FPLC fractions, an ELISA method was developed. Serially diluted FPLC fractions or human properdin standards (Quidel) were coated onto a 96-well ELISA plate overnight at $4^{\circ} \mathrm{C}$. After blocking with $1 \%$ BSA in PBS, plate-bound properdin was detected by incubation with a polyclonal goat anti-human properdin $\mathrm{Ab}(5 \mu \mathrm{g} / \mathrm{ml}$; Comp Tech), followed by incubation with horseradish peroxidase-conjugated rabbit anti-goat IgG (1:4000 dilution; Bio-Rad). The result of this ELISA assay was consistent with calculations based on OD280 measurement (using the extinction coefficient $120,200 \mathrm{M}^{-1} \mathrm{~cm}^{-1}$, determined by the ProtParam tool; http://www.expasy.org/tools/protparam.html) and typically yielded 200-300 $\mu \mathrm{g}$ mouse properdin from $50 \mathrm{ml}$ plasma as a starting material.

Production of rabbit anti-mouse properdin Abs. A polyclonal rabbit antimouse properdin $\mathrm{Ab}$ was generated by Cocalico using purified mouse properdin as an immunogen $(450 \mu \mathrm{g} / \mathrm{rabbit}$ in 6 immunizations $)$. Reactivity of the antiserum was tested by Western blot analysis of WT or $C \mathrm{fp}^{-/-}$serum. Function-blocking property of the antiserum was tested by preincubating protein A affinity column-purified IgG or nonimmune (i.e., control) rabbit IgG (Sigma-Aldrich) with WT serum ( $120 \mu \mathrm{g}$ IgG to $30 \mu \mathrm{l}$ mouse serum) for 60 minutes at $4{ }^{\circ} \mathrm{C}$, then assaying LPS-induced AP complement activity. To generate anti-mouse properdin $\operatorname{IgG~F}\left(\mathrm{ab}^{\prime}\right)_{2}$ fragments, $32 \mathrm{mg}$ protein $\mathrm{A}$ affinity column-purified anti-mouse properdin IgG or control rabbit IgG was dialyzed against $0.2 \mathrm{M}$ acetate buffer, $\mathrm{pH}$ 4.0. The dialyzed samples were then mixed with equal volumes of freshly prepared pepsin (SigmaAldrich) solution $(0.1 \mathrm{mg} / \mathrm{ml}$ in dialysis buffer). After incubating for 12 hours at $37^{\circ} \mathrm{C}$, the reaction was stopped by raising the $\mathrm{pH}$ to 8.0 using $2 \mathrm{M}$ Tris, $\mathrm{pH}$ 8.0. $\mathrm{F}\left(\mathrm{ab}^{\prime}\right)_{2}$ fragments from the reaction mixture were purified by Superose 12 column chromatography and confirmed by molecular weights on SDS-PAGE analysis.

Real-time PCR analysis of properdin expression. Single-cell suspension of mouse splenocytes was prepared as previously described (52). CD11 b+, $\mathrm{CD} 19^{+}$, and $\mathrm{CD} 90^{+}$cell populations were purified by magnetic beads (Miltenyi Biotech) or FACS method (52). Total RNAs were isolated from $\mathrm{BM}$ cells or splenocytes using TRIzoL reagent (Invitrogen). For real-time
PCR analysis of Cfp mRNA, the Taqman 1-step RT-PCR mix reagent kit and a 7300 real-time PCR system (both from Applied Biosystems) were used. Relative levels of mouse C $f p$ mRNA were calculated using Gapdb as a control ( $\Delta \Delta \mathrm{Ct}$ method).

Western blot analysis. Mouse serum $(2 \mu \mathrm{l})$ or PEG-precipitated total serum protein (from $12.5 \mu \mathrm{l}, 5 \% \mathrm{PEG}$ ) was boiled in sample buffer and loaded onto each lane of a $10 \%$ SDS-PAGE gel under reducing conditions. The separated proteins were blotted onto a PVDF membrane, which was then probed with rabbit anti-mouse properdin $\operatorname{IgG}(2 \mu \mathrm{g} / \mathrm{ml})$ for 1 hour, followed by detection with HRP-conjugated goat anti-rabbit IgG (1:4000 dilution; Bio-Rad).

Serum complement activity assays. 96-well ELISA plates were coated with LPS for AP complement activation assay, as described previously (21). Diluted mouse serum $(50 \mu \mathrm{l} /$ well $)$ was incubated on plates at $37^{\circ} \mathrm{C}$ for 1 hour, followed by detection of plate-bound activated C 3 using HRP-conjugated goat anti-mouse $\mathrm{C} 3 \mathrm{Ab}$ (1:4,000 dilution). AP complement activity was assayed in $\mathrm{Mg}^{++}$-EGTA $\mathrm{GVB}^{++}$buffer.

Statistics. Data were analyzed by 2-tailed Student's $t$ test, Wilcoxon/ Kruskal-Wallis test, or $\chi^{2}$ test as indicated. A $P$ value less than 0.05 was considered statistically significant. Data are presented as mean \pm SD unless otherwise indicated.

\section{Acknowledgments}

We thank Hector Molina for $\mathrm{Crry}^{+/-}$mice, Diane Mathis for KRN TCR transgenic mice, Andrew Cucchiara and Manolis Papasavvas for help with statistical analysis, and Alexis Ogdie for help with histopathological analysis. This work was supported by NIH grants AI85596, AI44970, AI49344, and AI63288 (to W.-C. Song) and a postdoctoral fellowship from the Arthritis Foundation (to Y. Kimura).

Received for publication November 18, 2009, and accepted in revised form July 14, 2010.

Address correspondence to: Wen-Chao Song, University of Pennsylvania School of Medicine, Room 1254 BRBII/III, 421 Curie Blvd., Philadelphia, Pennsylvania 19104, USA. Phone: 215.573.6641; Fax: 215.746.8941; E-mail: songwe@upenn.edu.
1. Walport MJ. Complement. First of two parts. NEngl J Med. 2001;344(14):1058-1066.

2.Zipfel PF, Skerka C. Complement regulators and inhibitory proteins. Nat Rev Immunol. 2009; 9(10):729-740.

3. Muller-Eberhard HJ. Molecular organization and function of the complement system. Annu Rev Biochem. 1988;57:321-347.

4. Pangburn MK, Schreiber RD, Muller-Eberhard HJ. Formation of the initial C3 convertase of the alternative complement pathway. Acquisition of C3b-like activities by spontaneous hydrolysis of the putative thioester in native C3. J Exp Med. 1981;154(3):856-867.

5. Harboe M, Mollnes TE. The alternative complement pathway revisited. J Cell Mol Med. 2008; 12(4):1074-1084.

6. Lutz HU, Jelezarova E. Complement amplification revisited. Mol Immunol. 2006;43(1-2):2-12.

7. Holers VM. The spectrum of complement alternative pathway-mediated diseases. Immunol Rev. 2008;223:300-316.

8. Parker C. Eculizumab for paroxysmal nocturnal haemoglobinuria. Lancet. 2009;373(9665):759-767.

9. Parker CJ, Kar S, Kirkpatrick P. Eculizumab. Nat Rev Drug Discov. 2007;6(7):515-516.

10. Ricklin D, Lambris JD. Complement-targeted therapeutics. Nat Biotechnol. 2007;25(11):1265-1275.

11. Pillemer L, Blum L, Lepow IH, Ross OA, Todd EW,
Wardlaw AC. The properdin system and immunity. I. Demonstration and isolation of a new serum protein, properdin, and its role in immune phenomena. Science. 1954;120(3112):279-285.

12. Pillemer L, Schoenberg MD, Blum L, Wurz L. Properdin system and immunity. II. Interaction of the properdin system with polysaccharides. Science. 1955;122(3169):545-549.

13. Muller-Eberhard HJ, Schreiber RD. Molecular biology and chemistry of the alternative pathway of complement. Adv Immunol. 1980;29:1-53.

14. Gotze O, Muller-Eberhard HJ. The alternative pathway of complement activation. Adv Immunol. 1976;24:1-35.

15. Fearon DT, Austen KF. Properdin: binding to C3b and stabilization of the C3b-dependent C3 convertase. J Exp Med. 1975;142(4):856-863.

16. Hourcade DE. Properdin and complement activation: a fresh perspective. Curr Drug Targets. 2008;9(2):158-164.

17. Hourcade DE. The role of properdin in the assembly of the alternative pathway $\mathrm{C} 3$ convertases of complement. J Biol Chem. 2006;281(4):2128-2132.

18. Spitzer D, Mitchell LM, Atkinson JP, Hourcade DE. Properdin can initiate complement activation by binding specific target surfaces and providing a platform for de novo convertase assembly. J Immunol. 2007;179(4):2600-2608.

19. Xu W, et al. Properdin binds to late apoptotic and necrotic cells independently of $\mathrm{C} 3 \mathrm{~b}$ and regulates alternative pathway complement activation. J Immunol. 2008;180(11):7613-7621.

20. Kemper C, Mitchell LM, Zhang L, Hourcade DE. The complement protein properdin binds apoptotic $T$ cells and promotes complement activation and phagocytosis. Proc Natl Acad Sci U S A. 2008;105(26):9023-9028.

21. Kimura Y, Miwa T, Zhou L, Song WC. Activatorspecific requirement of properdin in the initiation and amplification of the alternative pathway complement. Blood. 2008;111(2):732-740.

22. Gaarkeuken $\mathrm{H}$, et al. Complement activation by tubular cells is mediated by properdin binding. Am J Physiol Renal Physiol. 2008;295(5):F1397-F1403.

23. Xu C, Mao D, Holers VM, Palanca B, Cheng AM, Molina H. A critical role for murine complement regulator crry in fetomaternal tolerance. Science. 2000;287(5452):498-501.

24. Mao D, et al. Negligible role of antibodies and C5 in pregnancy loss associated exclusively with C3dependent mechanisms through complement alternative pathway. Immunity. 2003;19(6):813-822.

25. Li B, Sallee C, Dehoff M, Foley S, Molina H, Holers VM. Mouse Crry/p65. Characterization of monoclonal antibodies and the tissue distribution of a functional homologue of human MCP and DAF. J Immunol. 1993;151(8):4295-4305.

26. Kim YU, et al. Mouse complement regulatory pro- 
tein Crry/p65 uses the specific mechanisms of both human decay-accelerating factor and membrane cofactor protein. J Exp Med. 1995;181(1):151-159.

27. Ji $\mathrm{H}$, et al. Arthritis critically dependent on innate immune system players. Immunity. 2002 16(2):157-168

28. Matsumoto I, Staub A, Benoist C, Mathis D. Arthritis provoked by linked $\mathrm{T}$ and $\mathrm{B}$ cell recognition of a glycolytic enzyme. Science. 1999;286(5445):1732-1735.

29. Korganow AS, et al. From systemic T cell self-reactivity to organ-specific autoimmune disease via immunoglobulins. Immunity. 1999;10(4):451-461.

30. Schwaeble W, et al. Properdin, a positive regulator of complement activation, is expressed in human $\mathrm{T}$ cell lines and peripheral blood T cells. J Immunol. 1993;151(5):2521-2528.

31. Schwaeble W, et al. Expression of properdin in human monocytes. Eur J Biochem. 1994;219(3):759-764.

32. Wirthmueller U, et al. Properdin, a positive regulator of complement activation, is released from secondary granules of stimulated peripheral blood neutrophils. J Immunol. 1997;158(9):4444-4451.

33. Lakso $M$, et al. Efficient in vivo manipulation of mouse genomic sequences at the zygote stage. Proc Natl Acad Sci U S A. 1996;93(12):5860-5865.

34. Clausen BE, Burkhardt C, Reith W, Renkawitz $\mathrm{R}$, Forster I. Conditional gene targeting in macrophages and granulocytes using LysMcre mice. Transgenic Res. 1999;8(4):265-277.

35. Kemper C, Hourcade DE. Properdin: New roles in pattern recognition and target clearance. $\mathrm{Mol}$ Immunol. 2008;45(16):4048-4056.
36. Schwaeble WJ, Reid KB. Does properdin crosslink the cellular and the humoral immune response? Immunol Today. 1999;20(1):17-21.

37. Spath PJ, et al. Properdin deficiency in a large Swiss family: identification of a stop codon in the properdin gene, and association of meningococcal disease with lack of the IgG2 allotype marker G2m(n). Clin Exp Immunol. 1999;118(2):278-284.

38. Bathum L, et al. Association between combined properdin and mannose-binding lectin deficiency and infection with Neisseria meningitidis. Mol Immunol. 2006;43(5):473-479.

39. Rodriguez CI, et al. High-efficiency deleter mice show that FLPe is an alternative to Cre-loxP. Nat Genet. 2000;25(2):139-140.

40. Keller P, Tremml G, Rosti V, Bessler M. X inactivation and somatic cell selection rescue female mice carrying a Piga-null mutation. Proc Natl Acad Sci US A. 1999;96(13):7479-7483.

41. Miwa T, Zhou L, Kimura Y, Kim D, Bhandoola A, Song WC. Complement-dependent T-cell lymphopenia caused by thymocyte deletion of the membrane complement regulator Crry. Blood. 2009; 113(12):2684-2694.

42. Wu HJ, et al. Inflammatory arthritis can be reined in by CpG-induced DC-NK cell cross talk. J Exp Med. 2007;204(8):1911-1922.

43. Monach PA, Mathis D, Benoist C. The K/BxN arthritis model. Curr Protoc Immunol. 2010; Chapter 15:22.

44. Ji $\mathrm{H}$, et al. Genetic influences on the end-stage effector phase of arthritis. J Exp Med. 2001;194(3):321-330.
45. Kouskoff V, Korganow AS, Duchatelle V, Degott C, Benoist C, Mathis D. Organ-specific disease provoked by systemic autoimmunity. Cell. 1996;87(5):811-822.

46. Krenn V, Morawietz L, Haupl T, Neidel J, Petersen I, Konig A. Grading of chronic synovitis--a histopathological grading system for molecular and diagnostic pathology. Pathol Res Pract. 2002;198(5):317-325.

47. Krenn V, et al. Synovitis score: discrimination between chronic low-grade and high-grade synovitis. Histopathology. 2006;49(4):358-364.

48. Banda NK, Takahashi K, Wood AK, Holers VM, Arend WP. Pathogenic complement activation in collagen antibody-induced arthritis in mice requires amplification by the alternative pathway. IImmunol. 2007;179(6):4101-4109.

49. Kim DD, Miwa T, Song WC. Retrovirus-mediated over-expression of decay-accelerating factor rescues Crry-deficient erythrocytes from acute alternative pathway complement attack. J Immunol. 2006; 177(8):5558-5566.

50. Miwa T, et al. Decay-accelerating factor ameliorates systemic autoimmune disease in MRL/lpr mice via both complement-dependent and -independent mechanisms. Am J Pathol. 2007;170(4):1258-1266.

51. DiScipio RG. The fractionation of human plasma proteins. I. Affinity purification of human complement properdin. Protein Expr Purif. 1994;5(2):164-169.

52. Fang C, Zhang X, Miwa T, Song WC. Complement promotes the development of inflammatory T-helper 17 cells through synergistic interaction with Toll-like receptor signaling and interleukin-6 production. Blood. 2009;114(5):1005-1015. 